\title{
A Simulation Study on M/M/1 Queuing Model in a Medical Centre
}

\author{
S. Shanmugasundaram ${ }^{1}$, P. Umarani ${ }^{2}$ \\ ${ }^{1}$ Department of Mathematics, Government Arts college, Salem - 7, Tamilnadu, India \\ ${ }^{2}$ Department of Mathematics, AVS Engineering college, Salem - 3, Tamilnadu, India
}

\begin{abstract}
In this paper, we discuss the application of Simulation in M/M/1 queueing model in a medical centre. The simulation table provides a systematic method of studying the future behaviour of the system over time. The main aim of this paper is to find the waiting time of a patient in the queue, the waiting time of a patient in the medical centre, the idle time of a doctor, the queue length and also to compare with the mathematical solution.
\end{abstract}

Keywords: Inter - arrival Time, Service time, Waiting time, M/M/1 queueing model, Simulation, Queue length

\section{Introduction}

Waiting lines or Queues are familiar phenomena, which we observe quite frequently in our daily life. The study of a queueing problem would take into account: 1.The arrival distribution 2.The service distribution 3.The queue discipline 4.The number of service points 5.System capacity 6.Calling source [2].

Units arrive, at regular or irregular intervals of time, at a given point called the Service Centre. For example, ships arriving at a loading station, customers entering a department store, persons arriving at a cinema hall, letters arriving at a typist's desk, etc. All these units are called entries or arrivals of customers [4]. If the service station is free, the arriving customer will be served immediately; if not, the arriving customer will wait in line until the service is provided. Once service has been completed, the customer leaves the system. Whenever we have customers coming to a service facility in such a way that the customers have to wait, we have a queueing problem [8].

Queueing theory was introduced by A.K.Erlang in 1909. He published various articles about the study of jamming in telephone traffic [1]. The main aim of all investigations in queueing theory is to get the main performance measures of the system, which are the probabilistic properties like distribution function, density function, mean, variance of random variables. Number of customers in the system, number of waiting customers, consumption of the servers, response time of a customer, waiting time of a customer, Idle time of the server, busy time of a server can also be computed [5].

\section{M/M/1 Queuing Model}

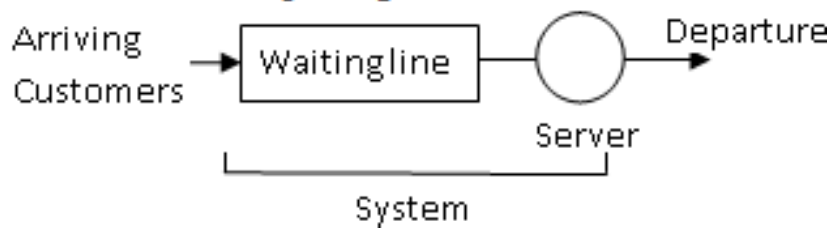

Simulation is a representation of reality through the use of a model or other device which will meet in the same manner as reality under a given set of conditions. Simulation has also been defined as the utilization of a system model that has the designed features of reality in order to produce the essence of actual operation [6]. Before introducing several simulations of queueing systems, it is necessary to understand the concepts of the system state, events and simulation clock. The state of the system is the number of units in the system and the status of the server, busy or idle. In a single channel queueing system, there are only two possible events that can change the state of the system. They are the arrival event and the departure event [7]. The arrival event occurs when a unit enters the system. The unit will find the server either idle or busy, therefore, either the unit starts service immediately, or the queue forms for the service. If the server is busy, the unit enters the queue. If the server is at rest and the queue is free, the unit begins service. It is not possible for the server to be idle while the queue is busy. After the completion of a service, the server either will become idle or will remain busy with the next unit [3]

Simulation clock times for arrivals and departures are computed in a simulation table customized for each problem. In simulation, events usually take place at random times, the randomness imitating uncertainty in real life. Random number also can be generated in Simulation. In a single channel queueing simulation, service times are generated from the distributions by using the random variables [10]

At a medical centre, there is one doctor who is to treat the patients. The patients arrival at the medical centre is a random phenomenon and the time between the arrivals varies from 6 a.m. to 12 p.m., and the service time varies from four minutes to thirty two minutes. The frequency distributions are given below.

Table 1: Arrival distribution

\begin{tabular}{|c|c|c|c|}
\hline S. No & Time & No of Patients & Probability \\
\hline 1 & $6-8$ & 4 & 0.008 \\
\hline 2 & $8-10$ & 37 & 0.076 \\
\hline 3 & $10-12$ & 76 & 0.157 \\
\hline 4 & $12-14$ & 91 & 0.188 \\
\hline 5 & $14-16$ & 48 & 0.099 \\
\hline 6 & $16-18$ & 63 & 0.130 \\
\hline 7 & $18-20$ & 107 & 0.221 \\
\hline 8 & $20-22$ & 54 & 0.111 \\
\hline 9 & $22-24$ & 5 & 0.010 \\
\hline Total & - & 485 & 1 \\
\hline
\end{tabular}




\section{International Journal of Science and Research (IJSR) \\ ISSN (Online): 2319-7064 \\ Index Copernicus Value (2013): 6.14 | Impact Factor (2014): 5.611}

Table 2: Service distribution

\begin{tabular}{|c|c|c|c|}
\hline S.No & Time & No of Patients & Probability \\
\hline 1 & $0-4$ & 5 & 0.077 \\
\hline 2 & $4-8$ & 7 & 0.108 \\
\hline 3 & $8-12$ & 15 & 0.231 \\
\hline 4 & $12-16$ & 13 & 0.202 \\
\hline 5 & $16-20$ & 12 & 0.185 \\
\hline 6 & $20-24$ & 7 & 0.117 \\
\hline 7 & $24-28$ & 3 & 0.050 \\
\hline 8 & $28-32$ & 2 & 0.030 \\
\hline Total & - & 65 & 1 \\
\hline
\end{tabular}

Table 4: Tag number table for service distribution

\begin{tabular}{|c|c|c|c|c|c|}
\hline S. No & Time & $\begin{array}{c}\text { No of } \\
\text { Patients }\end{array}$ & Probability & $\begin{array}{c}\text { Cumulative } \\
\text { Probability }\end{array}$ & Tag numbers \\
\hline 1 & $0-4$ & 5 & 0.077 & 0.077 & $0-76$ \\
\hline 2 & $4-8$ & 7 & 0.108 & 0.185 & $77-184$ \\
\hline 3 & $8-12$ & 15 & 0.231 & 0.416 & $185-415$ \\
\hline 4 & $12-16$ & 13 & 0.202 & 0.618 & $416-617$ \\
\hline 5 & $16-20$ & 12 & 0.185 & 0.803 & $618-802$ \\
\hline 6 & $20-24$ & 7 & 0.117 & 0.920 & $803-919$ \\
\hline 7 & $24-28$ & 3 & 0.050 & 0.970 & $920-969$ \\
\hline 8 & $28-32$ & 2 & 0.030 & 1 & $970-1000$ \\
\hline Total & - & 65 & 1 & - & - \\
\hline
\end{tabular}

Table 3: Tag number table for arrival distribution

\begin{tabular}{|c|c|c|c|c|c|}
\hline S. No & Time & $\begin{array}{c}\text { No of } \\
\text { Patients }\end{array}$ & Probability & $\begin{array}{c}\text { Cumulative } \\
\text { Probability }\end{array}$ & $\begin{array}{c}\text { Tag } \\
\text { numbers }\end{array}$ \\
\hline 1 & $6-8$ & 4 & 0.008 & 0.008 & $0-7$ \\
\hline 2 & $8-10$ & 37 & 0.076 & 0.084 & $8-83$ \\
\hline 3 & $10-12$ & 76 & 0.157 & 0.241 & $84-240$ \\
\hline 4 & $12-14$ & 91 & 0.188 & 0.429 & $241-428$ \\
\hline 5 & $14-16$ & 48 & 0.099 & 0.528 & $429-527$ \\
\hline 6 & $16-18$ & 63 & 0.130 & 0.658 & $528-657$ \\
\hline 7 & $18-20$ & 107 & 0.221 & 0.879 & $658-878$ \\
\hline 8 & $20-22$ & 54 & 0.111 & 0.990 & $879-989$ \\
\hline 9 & $22-24$ & 5 & 0.010 & 1 & $990-1000$ \\
\hline Total & - & 485 & 1 & - & - \\
\hline
\end{tabular}

Table 5: Simulation table

\begin{tabular}{|c|c|c|c|c|c|c|c|c|c|c|c|}
\hline S.No & $\begin{array}{l}\text { Random } \\
\text { number }\end{array}$ & \begin{tabular}{|c|} 
Inter arrival \\
time (min)
\end{tabular} & $\begin{array}{l}\text { Arrival } \\
\text { time }\end{array}$ & $\begin{array}{c}\text { Random } \\
\text { number }\end{array}$ & $\begin{array}{l}\text { Service } \\
\text { begins }\end{array}$ & $\begin{array}{c}\text { Service } \\
\text { Time (min) }\end{array}$ & $\begin{array}{l}\text { Service } \\
\text { ends }\end{array}$ & $\begin{array}{c}\text { Waiting time } \\
\text { in Queue } \\
\text { (min) }\end{array}$ & $\begin{array}{l}\text { Waiting time } \\
\text { in Medical } \\
\text { Centre (min) }\end{array}$ & $\begin{array}{c}\text { Idle Time } \\
\text { of Doctor } \\
\text { (min) }\end{array}$ & $\begin{array}{l}\text { Queue } \\
\text { length }\end{array}$ \\
\hline 1 & 483 & - & 6.00 & 837 & 6.00 & 6 & 6.06 & 0 & 6 & 0 & - \\
\hline 2 & 517 & 5 & 6.05 & 705 & 6.06 & 5 & 6.11 & 1 & 6 & 0 & 1 \\
\hline 3 & 063 & 2 & 6.07 & 068 & 6.11 & 1 & 6.12 & 4 & 5 & 0 & 1 \\
\hline 4 & 229 & 4 & 6.11 & 129 & 6.12 & 2 & 6.14 & 1 & 3 & 0 & 1 \\
\hline 5 & 797 & 7 & 6.18 & 597 & 6.18 & 4 & 6.22 & 0 & 4 & 4 & - \\
\hline 6 & 562 & 6 & 6.24 & 467 & 6.24 & 4 & 6.28 & 0 & 4 & 2 & - \\
\hline 7 & 066 & 2 & 6.26 & 543 & 6.28 & 4 & 6.32 & 2 & 6 & 0 & 1 \\
\hline 8 & 914 & 8 & 6.34 & 041 & 6.34 & 1 & 6.35 & 0 & 1 & 2 & - \\
\hline 9 & 511 & 5 & 6.39 & 513 & 6.39 & 4 & 6.43 & 0 & 4 & 4 & - \\
\hline 10 & 134 & 3 & 6.42 & 999 & 6.43 & 8 & 6.51 & 1 & 9 & 0 & 1 \\
\hline 11 & 657 & 6 & 6.48 & 840 & 6.51 & 6 & 6.57 & 3 & 9 & 0 & 1 \\
\hline 12 & 602 & 6 & 6.54 & 812 & 6.57 & 6 & 7.03 & 3 & 9 & 0 & 1 \\
\hline 13 & 511 & 5 & 6.59 & 153 & 7.03 & 2 & 7.05 & 4 & 6 & 0 & 1 \\
\hline 14 & 504 & 5 & 7.04 & 364 & 7.05 & 3 & 7.08 & 1 & 4 & 0 & 1 \\
\hline 15 & 132 & 3 & 7.07 & 126 & 7.08 & 2 & 7.10 & 1 & 3 & 0 & 1 \\
\hline 16 & 946 & 8 & 7.15 & 540 & 7.15 & 4 & 7.19 & 0 & 4 & 5 & - \\
\hline 17 & 579 & 6 & 7.21 & 978 & 7.21 & 8 & 7.29 & 0 & 8 & 2 & - \\
\hline 18 & 262 & 4 & 7.25 & 001 & 7.29 & 1 & 7.30 & 4 & 5 & 0 & 1 \\
\hline 19 & 787 & 7 & 7.32 & 494 & 7.32 & 4 & 7.36 & 0 & 4 & 2 & - \\
\hline 20 & 331 & 4 & 7.36 & 442 & 7.36 & 4 & 7.40 & 0 & 4 & 0 & - \\
\hline 21 & 600 & 6 & 7.42 & 135 & 7.42 & 2 & 7.44 & 0 & 2 & 2 & - \\
\hline 22 & 318 & 4 & 7.46 & 232 & 7.46 & 3 & 7.49 & 0 & 3 & 2 & - \\
\hline 23 & 151 & 3 & 7.49 & 457 & 7.49 & 4 & 7.53 & 0 & 4 & 0 & - \\
\hline 24 & 647 & 6 & 7.55 & 546 & 7.55 & 4 & 7.59 & 0 & 4 & 2 & - \\
\hline 25 & 890 & 8 & 8.03 & 248 & 8.03 & 3 & 8.06 & 0 & 3 & 4 & - \\
\hline 26 & 746 & 7 & 8.10 & 504 & 8.10 & 4 & 8.14 & 0 & 4 & 4 & - \\
\hline 27 & 995 & 9 & 8.19 & 292 & 8.19 & 3 & 8.22 & 0 & 3 & 5 & - \\
\hline 28 & 636 & 6 & 8.25 & 618 & 8.25 & 5 & 8.30 & 0 & 5 & 3 & - \\
\hline 29 & 589 & 6 & 8.31 & 220 & 8.31 & 3 & 8.34 & 0 & 3 & 1 & - \\
\hline 30 & 830 & 7 & 8.38 & 471 & 8.38 & 4 & 8.42 & 0 & 4 & 4 & - \\
\hline 31 & 444 & 5 & 8.43 & 627 & 8.43 & 5 & 8.48 & 0 & 5 & 1 & - \\
\hline 32 & 642 & 6 & 8.49 & 823 & 8.49 & 6 & 8.55 & 0 & 6 & 1 & - \\
\hline 33 & 590 & 6 & 8.55 & 527 & 8.55 & 4 & 8.59 & 0 & 4 & 0 & - \\
\hline 34 & 031 & 2 & 8.57 & 182 & 8.59 & 3 & 9.02 & 2 & 5 & 0 & 1 \\
\hline 35 & 590 & 6 & 9.03 & 638 & 9.03 & 5 & 9.08 & 0 & 5 & 1 & - \\
\hline
\end{tabular}

\section{Volume 5 Issue 3, March 2016}


International Journal of Science and Research (IJSR)

ISSN (Online): 2319-7064

Index Copernicus Value (2013): 6.14 | Impact Factor (2014): 5.611

\begin{tabular}{|c|c|c|c|c|c|c|c|c|c|c|c|}
\hline 36 & 304 & 4 & 9.07 & 147 & 9.08 & 2 & \begin{tabular}{|l|}
9.10 \\
\end{tabular} & 1 & 3 & 0 & 1 \\
\hline 37 & 169 & 3 & 9.10 & 320 & 9.10 & 3 & 9.13 & 0 & 3 & 0 & - \\
\hline 38 & 573 & 6 & 9.16 & 822 & 9.16 & 6 & $\begin{array}{l}9.22 \\
\end{array}$ & 0 & 6 & 3 & - \\
\hline 39 & 876 & 7 & 9.23 & 633 & 9.23 & 5 & 9.28 & 0 & 5 & 1 & - \\
\hline 40 & 216 & 3 & 9.26 & 548 & 9.28 & 4 & \begin{tabular}{|l}
9.32 \\
\end{tabular} & 2 & 6 & 0 & 1 \\
\hline 41 & 369 & 4 & 9.30 & 344 & 9.32 & 3 & \begin{tabular}{|l}
9.35 \\
\end{tabular} & 2 & 5 & 0 & 1 \\
\hline 42 & 607 & 6 & 9.36 & 461 & 9.36 & 4 & 9.40 & 0 & 4 & 1 & - \\
\hline 43 & 822 & 7 & 9.43 & 319 & 9.43 & 3 & 9.46 & 0 & 3 & 3 & - \\
\hline 44 & 918 & 8 & 9.51 & 246 & 9.51 & 3 & \begin{tabular}{|l}
9.54 \\
\end{tabular} & 0 & 3 & 5 & - \\
\hline 45 & 445 & 5 & 9.56 & 081 & 9.56 & 2 & \begin{tabular}{|l}
9.58 \\
\end{tabular} & 0 & 2 & 2 & - \\
\hline 46 & 658 & 7 & 10.03 & 195 & 10.03 & 3 & 10.06 & 0 & 3 & 5 & - \\
\hline 47 & 904 & 8 & 10.11 & 330 & 10.11 & 3 & 10.14 & 0 & 3 & 5 & - \\
\hline 48 & 686 & 7 & 10.18 & 649 & 10.18 & 5 & 10.23 & 0 & 5 & 4 & - \\
\hline 49 & 833 & 7 & 10.25 & 942 & 10.25 & 7 & 10.32 & 0 & 7 & 2 & - \\
\hline 50 & 490 & 5 & 10.30 & 091 & 10.32 & 2 & 10.34 & 2 & 4 & 0 & 1 \\
\hline 51 & 557 & 6 & 10.36 & 754 & 10.36 & 5 & \begin{tabular}{|l}
10.41 \\
\end{tabular} & 0 & 5 & 2 & - \\
\hline 52 & 626 & 6 & 10.42 & 737 & 10.42 & 5 & \begin{tabular}{|l|l}
10.47 \\
\end{tabular} & 0 & 5 & 1 & - \\
\hline 53 & 723 & 7 & 10.49 & 234 & 10.49 & 3 & $\begin{array}{l}10.52 \\
\end{array}$ & 0 & 3 & 2 & - \\
\hline 54 & 065 & 2 & 10.51 & 238 & 10.52 & 3 & 10.55 & 1 & 4 & 0 & 1 \\
\hline 55 & 469 & 5 & 10.56 & 222 & 10.56 & 3 & \begin{tabular}{|l}
10.59 \\
\end{tabular} & 0 & 3 & 1 & - \\
\hline 56 & 088 & 3 & 10.59 & 618 & 10.59 & 5 & 11.04 & 0 & 5 & 0 & - \\
\hline 57 & 148 & 3 & 11.02 & 173 & 11.04 & 2 & 11.06 & 2 & 4 & 0 & 1 \\
\hline 58 & 653 & 6 & 11.08 & 078 & 11.08 & 2 & 11.10 & 0 & 2 & 2 & - \\
\hline 59 & 920 & 8 & 11.16 & 830 & 11.16 & 6 & 11.22 & 0 & 6 & 6 & - \\
\hline 60 & 516 & 5 & 11.21 & 356 & 11.22 & 3 & 11.25 & 1 & 4 & 0 & 1 \\
\hline 61 & 244 & 4 & 11.25 & 870 & 11.25 & 6 & 11.31 & 0 & 6 & 0 & - \\
\hline 62 & 697 & 7 & 11.32 & 649 & 11.32 & 5 & \begin{tabular}{|l}
11.37 \\
\end{tabular} & 0 & 5 & 1 & - \\
\hline 63 & 896 & 8 & 11.40 & 609 & 11.40 & 4 & \begin{tabular}{|l}
11.44 \\
\end{tabular} & 0 & 4 & 3 & - \\
\hline 64 & 455 & 5 & 11.45 & 926 & 11.45 & 7 & 11.52 & 0 & 7 & 1 & - \\
\hline 65 & 718 & 7 & 11.52 & 741 & 11.52 & 5 & 11.57 & 0 & 5 & 0 & - \\
\hline 66 & 840 & 7 & 11.59 & 403 & 11.59 & 3 & 12.02 & 0 & 3 & 2 & - \\
\hline 67 & 259 & 4 & 12.03 & 189 & 12.03 & 3 & 12.06 & 0 & 3 & 1 & - \\
\hline 68 & 913 & 8 & 12.11 & 208 & 12.11 & 3 & 12.14 & 0 & 3 & 5 & - \\
\hline 69 & 741 & 7 & 12.18 & 666 & 12.18 & 5 & 12.23 & 0 & 5 & 4 & - \\
\hline 70 & 061 & 2 & 12.20 & 691 & 12.23 & 5 & 12.28 & 3 & 8 & 0 & 1 \\
\hline 71 & 498 & 5 & 12.25 & 254 & 12.28 & 3 & 12.31 & 3 & 6 & 0 & 1 \\
\hline 72 & 658 & 7 & 12.32 & 884 & 12.32 & 6 & 12.38 & 0 & 6 & 1 & - \\
\hline 73 & 549 & 6 & 12.38 & 456 & 12.38 & 4 & 12.42 & 0 & 4 & 0 & - \\
\hline 74 & 717 & 7 & 12.45 & 886 & 12.45 & 6 & 12.51 & 0 & 6 & 3 & - \\
\hline 75 & 501 & 5 & 12.50 & 276 & 12.51 & 3 & 12.54 & 1 & 4 & 0 & 1 \\
\hline 76 & 193 & 3 & 12.53 & 169 & 12.54 & 2 & 12.56 & 1 & 3 & 0 & 1 \\
\hline 77 & 826 & 7 & 1.00 & 966 & 1.00 & 7 & $\begin{array}{ll}1.07 \\
\end{array}$ & 0 & 7 & 4 & - \\
\hline 78 & 912 & 8 & 1.08 & 980 & 1.08 & 8 & \begin{tabular}{|l|}
1.16 \\
\end{tabular} & 0 & 8 & 1 & - \\
\hline 79 & 544 & 6 & 1.14 & 470 & 1.16 & 4 & $\begin{array}{l}1.20 \\
\end{array}$ & 2 & 6 & 0 & 1 \\
\hline 80 & 693 & 7 & 1.21 & 610 & 1.21 & 4 & 1.25 & 0 & 4 & 1 & - \\
\hline 81 & 907 & 8 & 1.29 & 101 & 1.29 & 2 & \begin{tabular}{|l}
1.31 \\
\end{tabular} & 0 & 2 & 4 & - \\
\hline 82 & 523 & 5 & 1.34 & 495 & 1.34 & 4 & \begin{tabular}{|l|}
1.38 \\
\end{tabular} & 0 & 4 & 3 & - \\
\hline 83 & 331 & 4 & 1.38 & 730 & 1.38 & 5 & 1.43 & 0 & 5 & 0 & - \\
\hline 84 & 925 & 8 & 1.46 & 006 & 1.46 & 1 & \begin{tabular}{|l|l}
1.47 \\
\end{tabular} & 0 & 1 & 3 & - \\
\hline 85 & 962 & 8 & 1.54 & 767 & 1.54 & 5 & \begin{tabular}{|l|}
1.59 \\
\end{tabular} & 0 & 5 & 7 & - \\
\hline 86 & 422 & 4 & 1.58 & 049 & 1.59 & 1 & 2.00 & 1 & 2 & 0 & 1 \\
\hline 87 & 657 & 6 & 2.04 & 856 & 2.04 & 6 & 2.10 & 0 & 6 & 4 & - \\
\hline 88 & 382 & 4 & 2.08 & 508 & 2.10 & 4 & 2.14 & 2 & 6 & 0 & 1 \\
\hline 89 & 147 & 3 & 2.11 & 012 & 2.14 & 1 & 2.15 & 3 & 4 & 0 & 1 \\
\hline 90 & 031 & 2 & 2.13 & 265 & 2.15 & 3 & 2.18 & 2 & 5 & 0 & 1 \\
\hline 91 & 135 & 3 & 2.16 & 470 & 2.18 & 4 & 2.22 & 2 & 6 & 0 & 1 \\
\hline 92 & 367 & 4 & 2.20 & 463 & 2.22 & 4 & \begin{tabular}{|l|}
2.26 \\
\end{tabular} & 2 & 6 & 0 & 1 \\
\hline 93 & 095 & 3 & 2.23 & 114 & 2.26 & 2 & 2.28 & 3 & 5 & 0 & 1 \\
\hline 94 & 121 & 3 & 2.26 & 294 & 2.28 & 3 & 2.31 & 2 & 5 & 0 & 1 \\
\hline 95 & 966 & 8 & 2.34 & 648 & 2.34 & 5 & 2.39 & 0 & 5 & 3 & - \\
\hline 96 & 056 & 2 & 2.36 & 089 & 2.39 & 2 & 2.41 & 3 & 5 & 0 & 1 \\
\hline 97 & 373 & 4 & 2.40 & 705 & 2.41 & 5 & 2.46 & 1 & 6 & 0 & 1 \\
\hline 98 & 534 & 6 & 2.46 & 543 & 2.46 & 4 & 2.50 & 0 & 4 & 0 & - \\
\hline 99 & 633 & 6 & 2.52 & 027 & 2.52 & 1 & 2.53 & 0 & 1 & 2 & - \\
\hline 100 & 314 & 4 & 2.56 & 768 & 2.56 & 5 & \begin{tabular}{|l|}
3.01 \\
\end{tabular} & 0 & 5 & 3 & - \\
\hline Total & - & 536 & & & - & 389 & - & 69 & 458 & 449 & 34 \\
\hline
\end{tabular}

\section{Volume 5 Issue 3, March 2016}




\section{International Journal of Science and Research (IJSR) \\ ISSN (Online): 2319-7064}

Index Copernicus Value (2013): 6.14 | Impact Factor (2014): 5.611

Simulation Calculation

Average Queue Length $=0.34$

Average Waiting Time of a Patient in Queue $=0.69$

Average Waiting Time of a Patient in a

Medical Centre $=4.58$

Arrival rate $=0.186$

Service rate $=0.257$

Idle Time of a Doctor $=4.49$

\section{Analytical Calculation $(\lambda<\mu)$}

Arrival rate $=0.01$

Service rate $=0.25$

Average Waiting Time of a patient in Queue $=0.132$

Average Waiting Time of a Patient in a

Medical Centre $=4.13$

Queue Length $=0.001$

\section{Numerical Study}

Table 6: Calculation table

\begin{tabular}{|c|c|c|}
\hline Categories & $\begin{array}{c}\text { Simulation } \\
\text { Calculation }\end{array}$ & $\begin{array}{c}\text { Mathematical } \\
\text { Calculation }\end{array}$ \\
\hline Arrival rate & 0.186 & 0.01 \\
\hline Service rate & 0.257 & 0.25 \\
\hline $\begin{array}{c}\text { Average Waiting Time of a } \\
\text { Patient in Queue }\end{array}$ & 0.69 & 0.132 \\
\hline $\begin{array}{c}\text { Average Waiting Time of a } \\
\text { Patient in a Medical Centre }\end{array}$ & 4.58 & 4.13 \\
\hline Queue Length & 0.34 & 0.001 \\
\hline
\end{tabular}

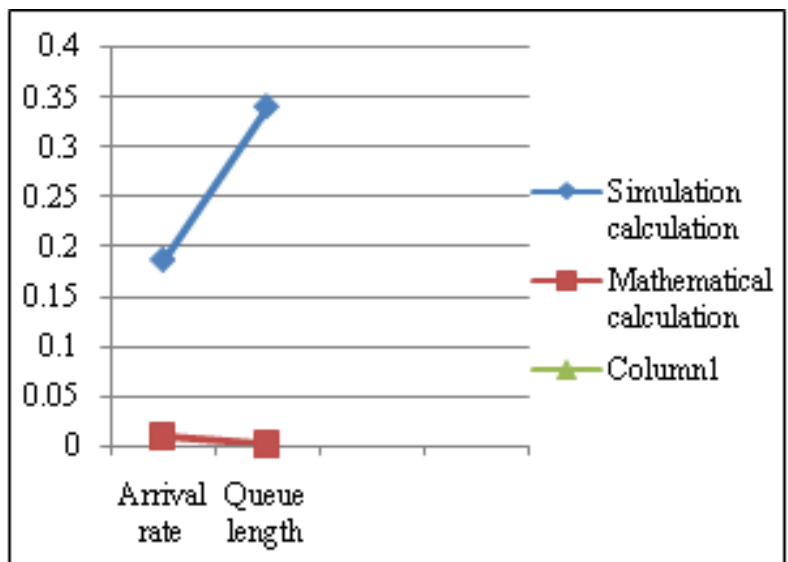

Figure 1: Comparison of Arrival rate with Queue length

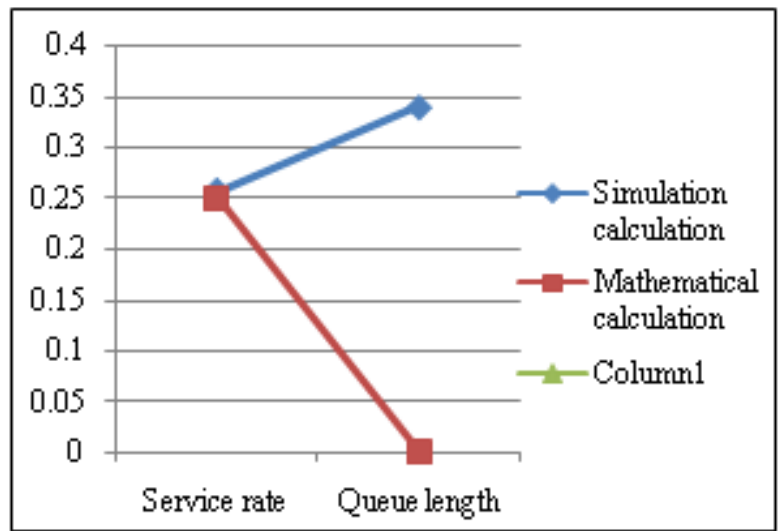

Figure 2: Comparison of Service rate with Queue length

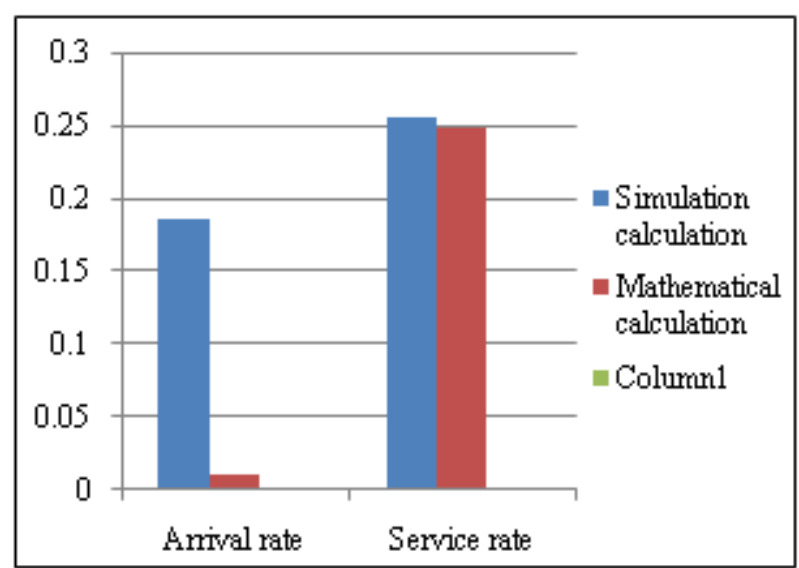

Figure 3: Comparison of Arrival rate with Service rate

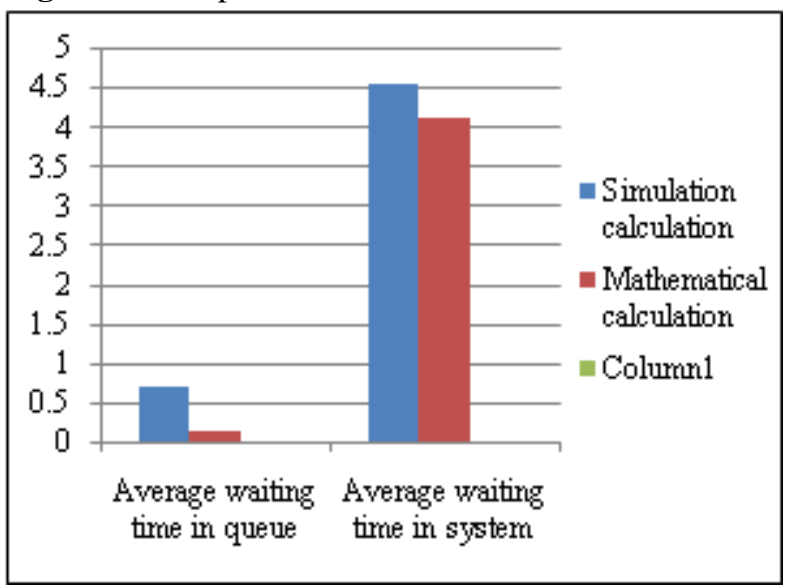

Figure 4: Comparison of waiting time of a patient in Queue and in medical centre

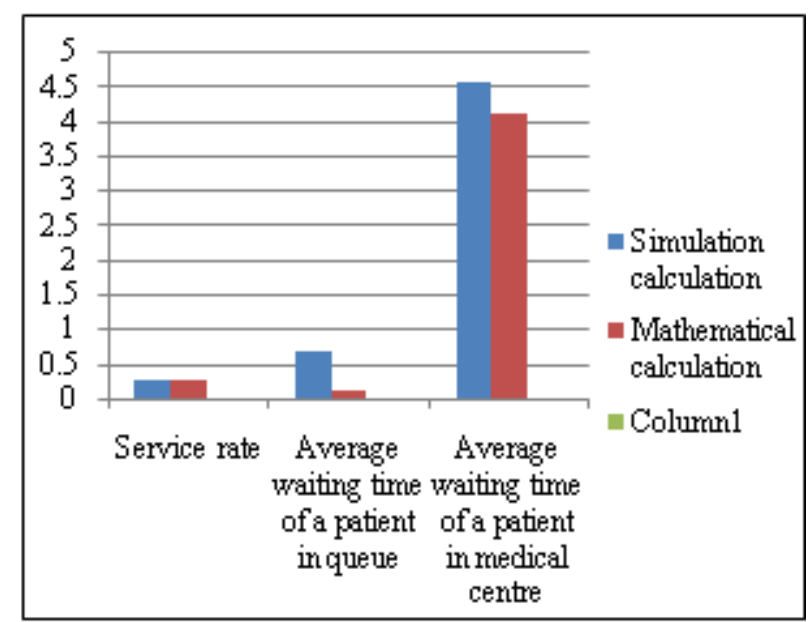

Figure 5: Comparison of Simulation Calculation with Mathematical Calculation

\section{Conclusion}

In this paper, we have studied the queueing system in simulation model of a medical centre assuming a single service station. In above discussion, we have calculated the queue length, average arrival time, average service time, idle time of a doctor, patient waiting time in the queue and in a medical centre. Also we have compared the calculation of simulation method and analytical method by using the bar diagram. This has given the feasibility of the system. The main purpose of this study is to develop an efficient 
procedure and to reduce the waiting time of the patients in a Medical Centre in future.

\section{References}

[1] Erlang,A.K (1909), "The Theory of Probabilities of Telephone Conversations", Nyt Jindsskriff Mathematic, B20, 33-39.

[2] Shanmugasundaram,S. and Punitha,S (2014), “A Simulation Study on Toll Gate System in M/M/1 Queueing Models", IOSR - Journal of Mathematics, e ISSN: 2278 - 5728, p - ISSN :2319 - 765X, Volume 10 , Issue 3.

[3] Wijewickrama,A.K.A (2006), "Simulation Analysis for reducing Queues in Mixed Patients' Outpatient Department", International Journal of Simulation Modelling, 5, 56 - 68.

[4] Shanmugasundaram,S. and Banumathi.P (2015), “A Study on Single Server Queue in Southern Railway using Monte Carlo Simulation”, Global Journal of Pure and Applied Mathematics, ISSN 0973 - 1768, Volume 11.

[5] Banks,J., Carson,J.S., Nelson,B.L., and Nicol,D.M(2001), "Discrete Event System Simulation", Prentice hall international series, third edition, 24 - 37.

[6] Sokolowski,J.A., Banks,C.M(2009), "Principals of Modelling and Simulation", Hoboken, NJ: Wiley.p.6, ISBN 978-0-470-28943-3.

[7] Kim,B.J(2011), "Conceptualization of Traffic Flow for Designing, Toll Plaza Configuration: A Case Study using Simulation with Estimated Traffic Volume", International Journal of Industrial Engineering, 18(1): 51 $-57$.

[8] Sundarapandian,V(2009), "7.Queueing Theory", Probability, Statistics and Queueing Theory, PHI learning, ISBN 8120338448.

[9] Little,J.D.C(1961), “A Proof for the Queueing Formula: $\mathrm{L}=\lambda \mathrm{W}$ ", Operations Research, Volume 9(3), pp.383 387, doi:10. 2307/167570.

[10] Laguna, $\mathrm{M}$ and Marklund,J(2005), "Business Process Modelling, Simulation and Design”, ISBN 0-13-091519$\mathrm{X}$, Pearson Prentice Hall.

\section{Author Profile}

Dr. S. Shanmugasundaram obtained M. Sc degree in Mathematics in 1986, M. Phil degree in 1988 and he obtained Ph.D degree in 2008. His area of specialization is "Applications of Stochastic Process in Queuing Models". He has 26 years of teaching experience in Mathematics. He has published ten Papers in national and international journals. He is working in the Department of Mathematics, Govt.Arts College, Salem -07, Tamil Nadu , India.

P. Umarani obtained M.Sc degree in Mathematics in 1999, M. Phil degree in 2004. Her area of specialization is "Queuing theory". She has 13 years of teaching experience in Mathematics. She is doing research in "Applications of Queuing theory". She has published one paper in international journal. She is working in the Department of Mathematics, AVS Engineering College, Salem - 03, Tamil Nadu,India. 